\title{
The effect of senior obstetric presence on maternal and neonatal outcomes in UK NHS maternity units
}

DOI:

10.1111/1471-0528.14649

\section{Document Version}

Accepted author manuscript

Link to publication record in Manchester Research Explorer

\section{Citation for published version (APA):}

Reid, H., Hayes, D., Wittkowski, A., Vause, S., Whitcombe, J., \& Heazell, A. (2017). The effect of senior obstetric presence on maternal and neonatal outcomes in UK NHS maternity units: A systematic review and meta-analysis. BJOG: an international journal of obstetrics and gynaecology . https://doi.org/10.1111/1471-0528.14649

\section{Published in:}

BJOG: an international journal of obstetrics and gynaecology

\section{Citing this paper}

Please note that where the full-text provided on Manchester Research Explorer is the Author Accepted Manuscript or Proof version this may differ from the final Published version. If citing, it is advised that you check and use the publisher's definitive version.

\section{General rights}

Copyright and moral rights for the publications made accessible in the Research Explorer are retained by the authors and/or other copyright owners and it is a condition of accessing publications that users recognise and abide by the legal requirements associated with these rights.

\section{Takedown policy}

If you believe that this document breaches copyright please refer to the University of Manchester's Takedown Procedures [http://man.ac.uk/04Y6Bo] or contact uml.scholarlycommunications@manchester.ac.uk providing relevant details, so we can investigate your claim.

\section{OPEN ACCESS}


1 Full title: The effect of senior obstetric presence on maternal and neonatal outcomes in

2 UK NHS maternity units: A systematic review and meta-analysis

3

4 Running title: A meta-analysis of the impact of senior obstetric presence

5

6

8

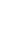

\section{Full Abstract}

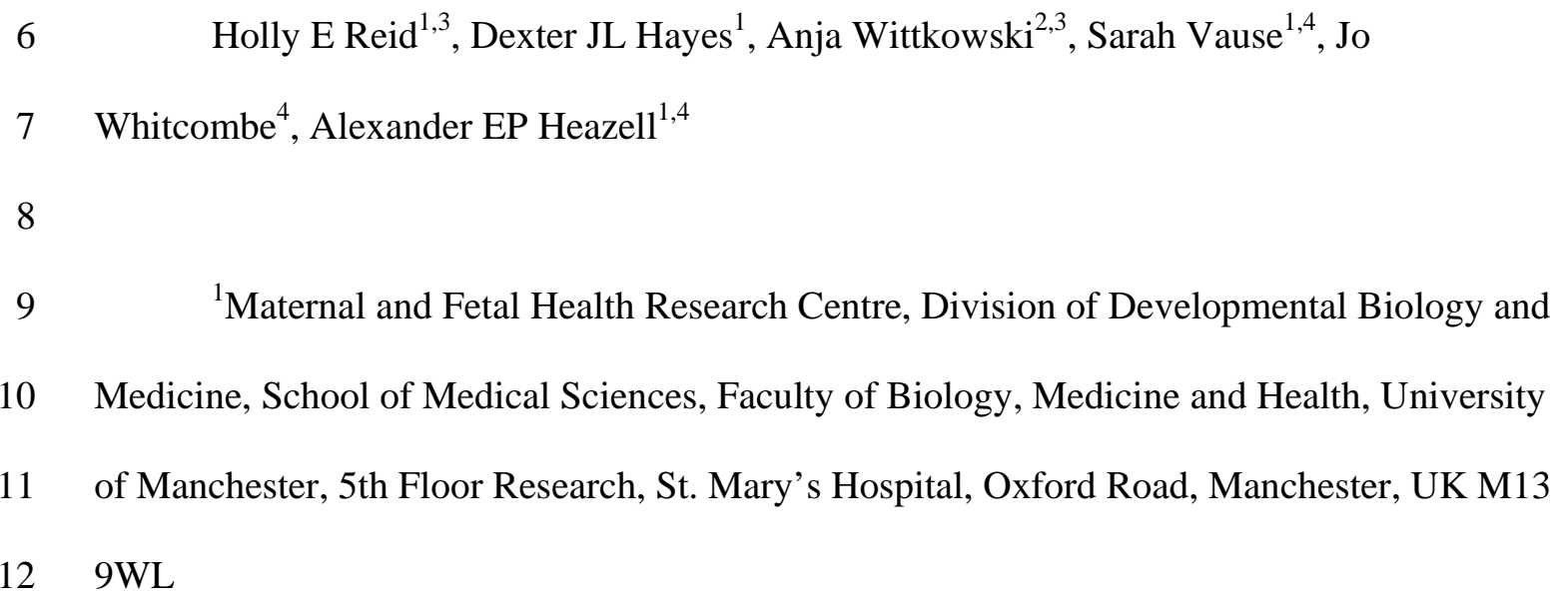




\section{Background}

27 There is little consensus regarding the hypothesised link between obstetric consultant

28 presence and maternal and neonatal outcomes.

\section{Objectives}

30 To pool existing data on the impact of consultant presence on the outcomes of women who

31 have given birth in UK NHS maternity units.

\section{Search strategy}

33 Twelve databases, grey literature and reference lists were searched.

\section{$34 \quad$ Selection criteria}

35 Studies conducted in UK NHS maternity units comparing outcomes during lesser consultant

36 presence versus increased consultant presence that reported mode of delivery and adverse

37 maternal or neonatal outcomes.

\section{Data collection and analysis}

39 Studies were divided into three groups by type of comparison: 1) hours of rostered consultant

40 presence during the weekend versus hours of rostered consultant presence during the week;

41 2) hours per week of rostered consultant presence pre-increase versus hours per week of

42 rostered consultant presence post-increase; 3) no rostered consultant presence versus rostered

43 consultant presence. A random effects meta-analysis was performed.

\section{Main results}

45 Fifteen studies fulfilled the inclusion criteria, presenting data from 125,856 births. Overall,

46 there was no significant difference between lesser and increased consultant presence for any

47 outcome. When data were stratified by comparison type, the likelihood of emergency

48 caesarean section was significantly lower (OR 0.91; 95\% CI 0.86-0.96) and non-instrumental

49 vaginal delivery was significantly higher (OR 1.07; 95\% CI 1.02-1.12) when rostered hours

50 of consultant presence per week were increased. 


\section{Conclusions}

52 Increased consultant presence has some effect on mode of delivery, but evidence for a benefit

53 for adverse outcomes was not identified.

54

55 Keywords

56 Obstetrics; meta-analysis; consultants; maternal outcomes; neonatal outcomes

57

58 Tweetable Abstract

59 Increasing hours of NHS obstetric consultant presence may increase chance of non-

60 instrumental vaginal delivery 


\section{Introduction}

62 Organisations in the UK have recommended increasing the number of hours

63 consultant obstetricians are present on the maternity unit. ${ }^{1-6}$ Consultant obstetricians, who

64 have completed all specialist training and examinations in obstetrics, have ultimate

65 responsibility for patients; equivalent to an attending physician in the USA. Recently,

66 guidelines have shifted focus to ensure consultant presence during working hours Monday to

67 Friday with an aim to extend this to seven days a week. ${ }^{7}$ The drivers for this increase in

68 consultant presence include rising birth rate, increasing case complexity and cost of litigation

69 claims. $^{8,9}$

70 However, these recommendations lack a robust evidence base to support the notion

71 that increased consultant presence translates into better outcomes. Observational studies have

72 evaluated outcomes for mothers and babies depending on time of birth. One such study in

73 Scotland found that the risk of neonatal death from intrapartum hypoxia was greater outside

74 the normal working week (5.6 per 10,000 births versus 4.2 per 10,000 births; odds ratio (OR)

75 1.30; 95\% CI 1.10-1.60). ${ }^{10}$ This could be explained by the level of staffing at different times

76 of day. However, another similar UK multi-centre study of 87,501 births found no difference

77 in neonatal morbidity, but demonstrated that obstetric intervention was less likely outside of

78 scheduled consultant presence. ${ }^{11}$ Critically, analysis of rotas or time periods rather than actual

79 consultant presence may overlook consultant presence when consultants have been called

80 in. ${ }^{12}$ The presumed link between consultant presence and an improvement in outcome is far

81 from established.

82 Nevertheless, many units have made significant moves towards fulfilment of the

83 Royal College of Obstetricians and Gynaecologists’ (RCOG) earlier recommendations ${ }^{3-6}$ to

84 increase the number of hours of consultant presence, ${ }^{13}$ albeit through different approaches,

85 with some units investigating the impact through observational studies. However, single unit 
86 studies are under-powered to identify differences in rare but serious outcomes. Amalgamation

87 of data would increase the ability to determine whether consultant presence affects perinatal

88 outcomes and understand sources of variation. A meta-analysis comparing the impact of

89 continuous resident consultant cover to other models of cover found that instrumental

90 deliveries decreased when consultants were resident. ${ }^{14}$ However, this meta-analysis only

91 included studies if they measured outcomes during a period of continuous consultant

92 presence and provided adequate information in the title and abstract. Furthermore, limits were

93 applied to study design, which resulted in only six included studies.

94 We conducted a systematic review and meta-analysis to compare and pool the effects of

95 increased consultant presence across NHS maternity units and understand the determinants of

96 these effects, using an updated and more inclusive search strategy than previously

97 employed. $^{14}$

$99 \underline{\text { Methods }}$

100 Protocol

101 The systematic review and meta-analysis were reported in accordance with the Meta-analysis

102 of Observational Studies in Epidemiology (MOOSE) guidelines. ${ }^{15}$ The review protocol was

103 registered with the International Prospective Register of Systematic Reviews (PROSPERO)

104 on 26 February 2016 (registration number CRD42016035455). After the search had been

105 completed it was apparent that inclusion of qualitative outcomes was not feasible because

106 very few studies included these outcomes, and often authors used a closed-question survey

107 and reported numeric results which did not allow for synthesis of qualitative findings.

108 Population studied and study inclusion criteria

109 Studies that examined women of any age who gave birth in a UK NHS maternity unit were

110 included as the RCOG recommendations were primarily intended for UK NHS units, not 
111 privately funded units or those abroad adopt different staffing models. We planned that this

112 would include secondary maternity units that offer routine and specialised care to women

113 with low-moderate-risk pregnancies and tertiary units that carry out highly specialised care in

114 addition to secondary care (although definitions of secondary and tertiary maternity units can

115 vary). The search only included studies reported in the English language because it was

116 assumed that studies conducted in UK NHS hospitals would be reported in English only.

117 There were no restrictions on date of publication or study design.

\section{Study exclusion criteria}

119 Any studies that focused on non-NHS maternity units or places of birth not normally attended

120 to by consultant obstetricians (e.g., midwifery-led unit or home) were excluded.

\section{Intervention}

123 Exposures of interest included any increase in obstetric consultant presence; thus, any

124 studies that involved a comparison of outcomes during lesser consultant presence versus

125 increased consultant presence were included (e.g., outcomes during a nightshift covered by a

126 registrar only with a consultant off-site versus a nightshift covered by a resident consultant).

\section{Outcome measures}

128 Outcomes of interest included emergency caesarean section rate, non-instrumental vaginal

129 delivery rate, instrumental delivery rate, stillbirth rate, neonatal death rate, perinatal mortality

130 rate, maternal death rate, and admission to a neonatal intensive care unit (NICU admission).

131 Mode of delivery was chosen as a primary outcome because this was the most reported

132 outcome in prior studies investigating consultant presence. Other secondary outcomes

133 included rate of postpartum haemorrhage $(\mathrm{PPH})$ and $3^{\text {rd }}$ and $4^{\text {th }}$ degree tears.

135 Information sources and search 
136 The literature searches were conducted by HR (a research assistant) and JW (a clinical

137 librarian) in EMBASE, MEDLINE, PsycINFO, CINAHL, Web of Science, Health

138 Management Information Consortium, Applied Social Sciences Index and Abstracts, and

139 Google Scholar. In order to uncover any relevant unpublished studies and grey literature the

140 Centre for Reviews and Dissemination databases, ProQuest Dissertations and Theses: UK

141 and Ireland: Health and Medicine, and EThOS were searched. Publications identified in the

142 searches were published between 1969 and 2016. Furthermore, reference lists of relevant

143 studies were also examined. See Appendix S1 for the EMBASE search strategy.

145 Study selection and data collection

146 Duplicates were removed and the most recent and complete version of the studies were

147 reviewed for eligibility. All relevant studies were assessed for eligibility by two reviewers

148 (HR and DH) independently according to the pre-specified inclusion and exclusion criteria.

149 A proforma was developed a priori for extracting the data from each study. The data

150 extraction was performed by $\mathrm{HR}$ and $\mathrm{DH}$ and if a disagreement occurred a third reviewer

151 (AH) was consulted to resolve the issue. In the event of missing data or identification of an

152 eligible abstract, authors were contacted by email, telephone, and post to obtain the

153 unpublished information in writing. If data were inconsistent, clarification was sought, or the

154 data were excluded from the analysis if the authors were uncontactable. All studies were

155 required to report at least one mode of delivery outcome, as well as the unit delivery volume

156 (average number of deliveries per year), the cohort sizes, the study design, the duration of the

157 study, and comparison hours of consultant presence.

158

159 Risk of bias 
160 The studies included in the review were subjected to a risk of bias assessment using the

161 Newcastle-Ottawa Quality Assessment Scale (NOS) ${ }^{16}$ by HR and DH independently. The

162 NOS, designed to assess the quality of non-randomised studies included in meta-analyses,

163 comprises of eight items categorised into themes of selection, comparability, and outcome. It

164 uses a nine-point rating system; where the higher the score the lower the risk of bias. The

165 selection criterion assesses the representativeness of the exposed cohort, selection of the non-

166 exposed cohort, how exposure was ascertained and the demonstration that the outcome(s) of

167 interest was not present prior to the study. The comparability criterion assesses the number of

168 variables controlled for, and the outcome criterion assesses how the outcome(s) of interest

169 was reported, whether the follow-up was long enough for the outcome(s) to occur and

170 whether all participants were accounted for. If a study's risk of bias was categorised as high

171 (score of six or fewer) ${ }^{17}$, the effect of removing this study from the meta-analysis was tested.

173 Statistical analyses

174 Meta-analysis was conducted using STATA (Version 14). ${ }^{18}$ Random effects meta-analysis 175 was performed in anticipation of heterogeneity between studies due to study design. The $\mathrm{I}^{2}$

176 statistic, derived from Cochran's chi-squared statistic $Q$, was calculated to describe the 177 percentage of between-study variation attributable to variability in the true exposure effect. ${ }^{19}$

178 Heterogeneity was classified as low $\left(\mathrm{I}^{2}=0-40 \%\right)$, moderate $\left(\mathrm{I}^{2}=30-60 \%\right)$, substantial $\left(\mathrm{I}^{2}=50\right.$ -

$17990 \%)$, or considerable $\left(\mathrm{I}^{2}=75-100 \%\right) .{ }^{20}$ Meta-regression was undertaken to test the effect of

180 unit delivery volume and the period of the study. All studies were categorised into three

181 groups depending on the comparison adopted: 1) hours of rostered consultant presence during

182 the weekend versus hours of rostered consultant presence during the week; 2) hours per week

183 of rostered consultant presence pre-increase versus hours per week of rostered consultant

184 presence post increase; and 3) no rostered consultant presence versus rostered consultant 
185 presence. Rostered consultant presence describes the period in which a consultant is

186 physically present and immediately available on the labour ward with no other duties. ${ }^{4}$ Forest

187 plots were constructed to show whether differences within the three groups or type of

188 maternity unit had any significant effect on each outcome. Funnel plots were created to test

189 for small-study effects.

190

191

\section{Results}

\section{Study characteristics}

Our systematic search strategy identified 412 titles (see Figure 1). After removal of

194 duplicates and screening of abstracts, 33 publications were fully evaluated. After removal of

195 publications that did not meet inclusion criteria and/or lacked relevant data, 15 titles that all

196 reported single-centre studies related to consultant presence and perinatal outcomes were

197 included in the final analysis. ${ }^{21-35}$ Thirteen studies reported births for all modes of delivery

198 (emergency caesarean sections, instrumental deliveries, and non-instrumental vaginal

199 deliveries), ${ }^{21-24,26,27,29-35}$ one study included elective caesarean sections and therefore data for

200 caesarean sections were excluded. ${ }^{25}$ Inductions of labour (IOLs) were only mentioned in six

201 studies (five included IOLs, ${ }^{22,25,26,33,34}$ one excluded IOLs ${ }^{35}$ ). Although, IOLs are more likely

202 to occur during 'office hours' and are directed to higher-risk pregnancies, we could not

203 conduct a sensitivity analysis due to the small number of studies providing information on

204 IOLs in their datasets. Another study reported both instrumental and non-instrumental vaginal

205 deliveries as a single outcome; ${ }^{28}$ thus, data for those outcomes were excluded. Ten studies

206 reported data for other outcomes, such as stillbirth, neonatal death, NICU admission, $3^{\text {rd }}$ and

$2074^{\text {th }}$ degree tears, and postpartum haemorrhage. Nine studies were conducted in secondary

208 maternity units and six were conducted in tertiary units (see Table S1 for study

209 characteristics). The majority of studies had a low risk of bias in the assessed domains, with 
210 the exception of the Fleming et al. ${ }^{25}$ study which was assessed as having a high risk of bias

211 (see Figure S1; Table S2). The following analyses were also conducted with the exclusion of

212 this study; however, this did not change the findings, therefore the meta-analysis results

213 presented include all studies.

215 Consultant presence and emergency caesarean section rate

216 All studies included in the analysis, except for one, ${ }^{25}$ excluded elective caesarean sections.

217 This was important because elective caesarean sections are more likely to occur during

218 'office hours' and carry a lower risk of adverse outcomes. The 14 studies that reported

219 emergency caesarean sections recorded the outcome of 119,397 births ( $94.9 \%$ of births in the

220 whole analysis). There was no significant difference in emergency caesarean section rates

221 between lesser consultant presence and increased consultant presence (OR 0.98; 95\% CI 0.92

222 to 1.05). Table 1.

223 There was substantial heterogeneity within the data $\left(\mathrm{I}^{2}=68.2 \%\right)$. Firstly, the data were

224 stratified by comparison group and inspection of the forest plot (see Figure 2) suggested that

225 the likelihood of emergency caesarean section was significantly lower during an increase in

226 rostered consultant presence hours per week versus pre-increase consultant presence (Group

227 2) (OR 0.91; 95\% CI 0.86 to 0.96). Secondly, when data were stratified by type of unit

228 (secondary or tertiary), inspection of the forest plot (see Figure S2) indicated that this did not

229 have a significant effect on emergency caesarean section rates.

230 Meta-regression was performed to search for any associations between unit delivery

231 volume and study period in months, which showed no evidence for an association of any

232 covariates with the size of the exposure effect $\left(\mathrm{R}^{2}=0.21\right)$. A contour enhanced funnel plot

233 demonstrated that small study effects did not have an influence on the significance of this 
234 result (see Figure S3; Harbord's test, $\mathrm{p}=0.74$ ); this is also supported by the similarity between

235 the output of random and fixed effects meta-analyses.

236

237 Consultant presence and non-instrumental vaginal delivery rate

238 The 14 studies that reported non-instrumental vaginal deliveries recorded the outcome of 239 117,684 births (93.5\% of births in the whole analysis). Overall, there was no significant

240 difference in non-instrumental vaginal deliveries between lesser consultant presence and 241 increased consultant presence (OR 1.00; 95\% CI 0.95 to 1.06). There was substantial 242 heterogeneity within the data $\left(\mathrm{I}^{2}=71.7 \%\right)$. Following inspection of the forest plot for data

243 stratified by comparison group (see Figure 3), non-instrumental vaginal deliveries were

244 significantly more likely to occur during increased hours per week of rostered consultant

245 presence when compared to pre-increase hours per week of rostered consultant presence

246 (Group 2) (OR 1.07; 95\% CI 1.02-1.12). When the data were stratified by type of unit, again

247 no significant difference was observed with regard to non-instrumental vaginal deliveries.

\section{Consultant presence and instrumental delivery rate}

250 The studies that reported instrumental delivery rates were identical to those that reported non-

251 instrumental vaginal deliveries (93.5\% of births in the whole analysis). Overall, there was no

252 significant difference in instrumental deliveries between lesser consultant presence and

253 increased consultant presence (OR 1.04; 95\% CI 0.98 to 1.10). There was moderate

254 heterogeneity within the data $\left(\mathrm{I}^{2}=46.0 \%\right)$. Stratification of the data by comparison group (see

255 Figure S4) and by type of unit did not demonstrate any differences.

257 Consultant presence and maternal and neonatal outcomes 
259 recorded the frequency of maternal death, Freites et al. ${ }^{26}$ reported one death and Mackie et

260 al. $^{29}$ reported two deaths; due to the low incidence they were not included in the analysis.

261 There were no significant differences in NICU admission, neonatal death, stillbirth, PPH or

262 tears. All studies that reported NICU admission were hospitals with a level 3 NICU, except

263 one unit which was level $2,^{33}$ and therefore data could not be stratified by level of NICU.

264 Outcomes showed heterogeneity ranging $\mathrm{I}^{2}=0.0-83.8 \%$.

\section{Discussion}

\section{Main findings}

The review consolidates research investigating the effect of consultant presence on

maternal and neonatal outcomes, but also identifies that three different methods of comparing

obstetric consultant presence in UK NHS maternity units have been employed yielding

different results. The meta-analysis of 15 studies found no overall significant difference

between prior levels of consultant presence and increased consultant presence with regards to

mode of delivery or maternal and neonatal outcomes. However, when data were stratified by comparison group, findings indicated that increased hours per week of rostered consultant presence significantly reduced the likelihood of emergency caesarean sections and significantly increased the likelihood of non-instrumental vaginal deliveries. Meta-regression indicated that unit delivery volume and study period were not associated with the exposure effect. Furthermore, two-thirds of studies were assessed as having a low risk of bias and removal of the study with the highest risk of bias ${ }^{25}$ did not change the results, suggesting the

280 quality of studies did not affect the findings. Overall, the data indicate that increasing consultant presence had an impact on the mode of delivery, rather than maternal and neonatal morbidity or mortality. 
284 Strengths and Limitations

285 This comprehensive review was strengthened by the use of a prospective protocol, 286 with specified eligibility criteria and adherence to published guidelines. Furthermore, the 287 analysis included a varied range of maternity units with regards to unit location, unit type, 288 and unit delivery volume, suggesting a population of high- and low-risk women, which 289 increases the generalisability of the findings. However, there were limitations that constrain 290 the conclusions of this meta-analysis. There was a lack of information available regarding 291 midwifery or anaesthetic staff during the studies, and whether their numbers and level of 292 experience changed in line with the changes to consultant cover. This presents a confounder 293 that could not be investigated fully. Some of the outcomes, such as late intrauterine fetal 294 death and NICU admissions, do not reflect intrapartum management; thus, the possible effect 295 of consultant presence is reduced. The most serious intrapartum outcomes, intrapartum 296 stillbirth and maternal death from direct causes, are rarely reported or are such a rare 297 occurrence that any analysis would have to be enormous (i.e. nationwide) to demonstrate a 298 statistically significant difference. Furthermore, we identified that the arbitrariness of 299 categorisation and labels played a role in this review. Firstly, there was no definitive method 300 or resource to help categorise maternity units as secondary or tertiary. Secondly, the career

301 labels themselves, 'consultant' and 'registrar', pose a limitation, as a consultant is not 302 necessarily better than an experienced trainee who is nearly a consultant. 303 Interpretation

The involvement of consultants in obstetric care is considered important in providing 306 safer intrapartum outcomes. ${ }^{3-6}$ The present study clarifies the currently conflicting results

307 regarding a link between obstetric consultant presence and maternal and neonatal outcomes. 
308 The findings suggest that increasing rostered consultant presence would reduce emergency

309 caesarean section rates and increase non-instrumental vaginal deliveries. This would have a

310 number of benefits including reduced recovery time postpartum, reduced risk of maternal

311 infection and thrombosis, and reduced risk of neonatal complications. ${ }^{36}$ Although the reasons

312 for the reduction in caesarean section rate during periods of consultant presence in this meta-

313 analysis are not clear, consultant presence may increase junior doctors' clinical judgement,

314 confidence, and skills to allow a woman to give birth vaginally rather than performing an

315 emergency caesarean section prematurely. The reduction in caesarean sections did not seem

316 to be associated with instrumental birth. The present study found no difference in

317 instrumental vaginal delivery, which was not in agreement with previous observations that

318 women who gave birth during periods of no rostered consultant presence were less likely to

319 have an instrumental delivery. These contradictory observations may reflect controversy

320 surrounding certain instrumental procedures (e.g., rotation of the fetal head) $)^{37,38}$ and

321 variability between trainee doctors in performing instrumental deliveries, ${ }^{39}$ exerting a greater

322 influence over rates than consultant presence alone.

323 We did not observe an effect of obstetric consultant presence on NICU admissions or

324 stillbirths. One possible explanation is that senior input in labour is not a preventative

325 measure in the majority of these occurrences. NICU admissions may include very preterm

326 babies or babies with known structural anomalies that cannot be improved by an obstetric

327 consultant, and $86 \%$ of stillbirths occur prior to the onset of labour. ${ }^{40}$ The results also

328 demonstrated no effect on the other outcomes of PPH, neonatal death, and $3^{\text {rd }}$ and $4^{\text {th }}$ degree

329 tears. The most likely reason for this would be lack of data; despite combining studies within

330 a meta-analysis, the cumulative data are still under-powered to detect the frequency of rare

331 but serious events such as neonatal death due to intrapartum anoxia. Using previously

332 reported incidences, ${ }^{10}$ over 392,000 women would be required in each group to detect a 
333 statistically significant difference $(\mathrm{p}<0.05)$ in neonatal death due to intrapartum anoxia with $33480 \%$ power.

335 Statistically significant differences were only found for studies of increased hours per 336 week of rostered consultant presence (Group 2). A number of reasons may explain why this 337 statistically significant difference was found in the stratified analyses only. Firstly, this may 338 reflect statistical power as studies in Group 2 included a greater number of births. Secondly, 339 studies from Groups 1 and 3 compared rostered consultant presence during time of the week 340 and specific shifts, respectively. These comparisons rely on the assumption that consultants 341 are not present when they are on-call, however in an emergency situation the consultants 342 would be called in. This may have resulted in consultants being present on the ward during 343 times of no rostered consultant presence and this may explain why consultant presence was

344 not seen to impact any outcomes in these groups. ${ }^{12}$ Alternatively, there may be other 345 interventions introduced concurrently with increased consultant presence which also affect 346 mode of delivery, including increase of midwife or anaesthetist presence. For example in one 347 included study, the midwife to antenatal patient ratio increased from 1:35 to 1:28 due to 348 amalgamation of units, ${ }^{29}$ which could contribute to the increased likelihood of a non349 instrumental vaginal delivery. ${ }^{41}$ Another possibility could be increased cohesiveness of the 350 obstetric and midwifery team, which could result in stronger working relationships and better 351 management of the team by consultants, which offers another possibility for the different

352 findings observed in the overall analysis and the stratified analyses. To contextualise our 353 findings we attempted to find international studies comparing the effects of increased senior 354 obstetric presence on perinatal outcome, but we could find no directly comparable studies. 355 Consultant presence may deliver a cost saving because non-instrumental vaginal 356 deliveries cost considerably less than caesarean sections. ${ }^{42}$ However, our findings also imply 357 that consultant presence may not reduce maternal and neonatal mortality and morbidity, the 
358 predominant cause of medicolegal cases (which totalled £3.1 billion between 2000-2010) ${ }^{43}$, a

359 number of issues must be considered by healthcare-providers and policy-makers when

360 increasing consultant presence, including the financial repercussions (to employ a full-time

361 consultant for one year costs approximately £125,000). Further large scale studies, which can

362 adequately assess the frequency of these rare and serious outcomes, are needed to evaluate

363 the cost-effectiveness of increased consultant presence. Numerical analysis should also be

364 supported by other methodologies, such as Confidential Enquiries, ${ }^{44}$ to determine whether

365 consultant presence would have made a difference to perinatal outcome.

366

367 Conclusion

368 In conclusion, this meta-analysis suggests that consultant presence reduced the

369 likelihood of emergency caesarean sections and increased non-instrumental vaginal

370 deliveries. The findings shed some light on the relationship between obstetric consultant

371 presence and perinatal outcomes in UK NHS maternity units, providing further evidence that

372 consultant presence has an effect but evidence for an effect on more serious outcome

373 including mortality and morbidity was not identified.

374

375 Acknowledgements

376 We would like to thank the study authors who provided additional information regarding

377 their research to aid this meta-analysis.

\section{$378 \quad \underline{\text { Disclosure of interests }}$}

379 All authors have completed the ICMJE uniform disclosure form at

380 www.icmje.org/coi_disclosure.pdf and declare: no support from any organisation for the

381 submitted work; no financial relationships with any organisations that might have an interest

382 in the submitted work in the previous three years; no other relationships or activities that 
383 could appear to have influenced the submitted work. The ICMJE disclosure forms are

384 available as online supporting information.

385 Contribution to authorship

386 All authors had full access to all of the data and can take responsibility for the integrity of the

387 data and accuracy of the data analysis. HR, DH, AW, SV, and AH contributed to the study

388 concept and design. HR and AH wrote the protocol. HR and JW developed the search

389 strategy. HR, DH, SV, and JW acquired the data for the study. HR, DH, and AH contributed

390 to the analysis and interpretation of the data. HR developed the first draft of the manuscript

391 and DH, AW, SV, JW, and AH revised all manuscript drafts and approved the final version.

392 HR is the study guarantor.

393 Details of ethical approval

394 Ethical approval was not required because the systematic review did not involve human or

395 animal subjects, nor did it involve collecting data from patients’ medical records.

396 Patient Involvement

397 Patients were not involved in the design or conduct of this study.

398 Funding

399 The study was funded by Central Manchester University Hospitals NHS Foundation Trust

400 Charity. The funding source had no role in study design, data collection, data analysis, data

401 interpretation or preparation of the manuscript. The corresponding author had full access to

402 all the data in the study and had final responsibility for the decision to submit for publication.

403 Transparency

404 The lead author (HR) affirms that the manuscript is an honest, accurate, and transparent

405 account of the study being reported. No important aspect of the study has been omitted and

406 discrepancies from the registered protocol have been explained.

$407 \quad$ Data sharing 
408 Additional data and the study reports can be obtained from the corresponding author on 409 request.

\section{References}

415 1. Healthcare Commission. Towards better births: a review of maternity services in England. London: Commission for Healthcare Audit and Inspection, 2008.

2. National Health Service Litigation Authority. Clinical Negligence Scheme for Trusts.

4. Royal College of Obstetricians and Gynaecologists, Royal College of Midwives, Royal College of Obstetricians and Gynaecologists, 2010. 
7. Royal College of Obstetricians and Gynaecologists. Providing quality care for women: Obstetrics and gynaecology workforce. London: Royal College of Obstetricians and Gynaecologists, 2016.

8. Sandall J, Homer C, Sadler E, Rudsill C, Bourgeault IL, Bewley S, et al. Staffing in maternity units: getting the right people in the right place at the right time. London: The King’s Fund, 2011.

9. National Audit Office. Maternity service in England. London: National Audit Office, 2013.

10. Pasupathy D, Wood AM, Pell JP, Mechan H, Fleming M, Smith GCS. Time of birth and risk of neonatal death at term: retrospective cohort study. BMJ. 2010; 341: c3498. doi: 10.1136/bmj.c3498

11. Knight HE, van der Meulen JH, Gurol-Urganci I, Smith CG, Kiran A, Thornton S, et al. Birth "Out-of-Hours”: an evaluation of obstetric practice and outcome according to the presence of senior obstetricians on the labour ward. PLoS Med. 2016; 13: e1002000. doi:10.1371/journal.pmed.1002000

12. Myers JE, Johnstone ED. Is there evidence of poorer birth outcomes for mothers and babies when the most senior obstetrician is not on site? PLoS Med. 2016; 13: e1002001. doi: 10.1371/journal.pmed.1002001

13. Royal College of Obstetricians and Gynaecologists. RCOG Census Report 2013. London: Royal College of Obstetricians and Gynaecologists, 2015.

14. Knight M, Henderson J, Kurinczuk JJ. Evidence review to support the National Maternity Review 2015; Report 3: systematic review and case studies to assess models of consultant resident cover and the outcomes of intrapartum care; and two international case studies of the delivery of maternity care. Oxford: National Perinatal Epidemiology Unit, University of Oxford, 2015. 
15. Stroup DF, Berlin JA, Morton SC, Ingram Olkin G, Williamson D, Rennie D, et al. Meta-analysis of observational studies in epidemiology (MOOSE): a proposal for reporting. Meta-analysis Of Observational Studies in Epidemiology (MOOSE) Group. JAMA. 2000; 283: 2008-12. doi: 10.1001/jama.283.15.2008

16. Wells GA, Shea B, O’Connell D, Peterson J, Welch V, Losos M, et al. The Newcastle-Ottawa Scale (NOS) for assessing the quality of nonrandomised studies in meta-analyses [Internet]. 2014 [cited $1^{\text {st }}$ April 2016]. Available from: http://www.ohri.ca/programs/clinical_epidemiology/oxford.asp.

17. Lo CK, Mertz D, Loeb M. Newcastle-Ottawa scale: Comparing reviewers’ to authors’ assessments. BMC Med Res Methodol. 2014; 14: 45. doi: 10.1186/1471-2288-14-45

18. StataCorp. STATA Statistical Software, release 14. College Station, TX: StataCorp LP, 2015.

19. Higgins JPT, Thompson SG, Deeks JJ, Altman DG. Measuring inconsistency in metaanalyses. BMJ. 2003; 327: 557-60. doi: 10.1136/bmj.327.7414.557

20. Deeks JJ, Higgins JPT, Altman DG. Chapter 9: analysing data and undertaking metaanalyses [Internet]. In: Higgins JPT, Green S, eds. Cochrane Handbook for Systematic Reviews of Interventions. 2011 [cited $17^{\text {th }}$ June 2016]. Available from: www.cochrane-handbook.org.

21. Ahmed I, Wyldes MP, Chong HP, Barber KJ, Tan BK. 24/7 consultant presence in a UK NHS tertiary maternity unit. Lancet. 2015; 386: 951-2. doi: 10.1016/S0140$\underline{6736(15) 00058-6}$

22. Aiken CE, Aiken AR, Scott JG, Brockelsby JT. Weekend working: a retrospective cohort study of maternal and neonatal outcomes in a large NHS delivery unit. Eur $J$ Obstet Gynecol Reprod Biol. 2016; 199: 5-10. doi: 10.1016/j.ejogrb.2016.01.034 
23. Albury C, Ashelby L, Basude S. Resident consultant on-call: 6 month evaluation of service. Does resident consultant presence change outcomes in a tertiary maternity unit? BJOG. 2015; 122(S2): 78-9. doi: 10.1111/1471-0528.13387

24. Ballal A, Dawood F, Scholefield H. Resident consultant versus on call cover on delivery suite: are outcomes better? Arch Dis Child Fetal Neonatal Ed. 2012; 97(S1): A78. doi: 10.1136/fetalneonatal-2012-301809.256

25. Fleming A, Martindale EA, Schram CMH. Reducing caesarean section rates through choice and collaboration. Arch Dis Child Fetal Neonatal Ed. 2013; 98(S1): A55-A56. doi: 10.1136/archdischild-2013-303966.189

26. Freites J, Ruprai C, Paul H, Lindow SW. Resident consultant presence in labour ward after midnight - a retrospective cohort study of 5318 deliveries. J Perinat Med. 2012; 40: 615-8. doi: 10.1515/jpm-2012-0060

27. Katakam N, Chan D, Rockett T, Wong S, Das S. Does consultant obstetrician presence on the labour ward improve quality of patient care? A review of quality indicators at Royal Bolton Hospital. Arch Dis Child Fetal Neonatal Ed. 2012; 97(S1): A111. doi: 10.1136/fetalneonatal-2012-301809.362

28. Khalil A, Suff N, Blott M, O’Brien P. Does the presence of a consultant on the delivery suite reduce the emergency caesarean section rate? Arch Dis Child Fetal Neonatal Ed. 2011; 96(S1): Fa10. doi: 10.1136/adc.2011.300160.30

29. Mackie FL, Afadapa F, Moise J, Amu O. Maternal and neonatal outcomes after the amalgamation of two maternity units and consequent increased consultant labour ward presence: a retrospective population-cased study. Arch Dis Child Fetal Neonatal Ed. 2014; 99(S1): A21-A22. doi: 10.1136/archdischild-2014-306576.60 
30. Nunes N, Rogers C, Johnston T. Impact of overnight consultant presence - outcomes and attitudes. Arch Dis Child Fetal Neonatal Ed. 2014; 99(S1): A22. doi: 10.1136/archdischild-2014-306576.62

31. Rajesh U, Merrick K. Impact of resident consultants on call at night in York Teaching Hospital. Arch Dis Child Fetal Neonatal Ed. 2014; 99(S1): A28. doi: 10.1136/archdischild-2014-306576.79

32. Siddiqui F, Green A, Moore J, Kean L. Resident obstetric consultant cover: does it make a difference to vaginal delivery rates or perinatal morbidity? Arch Dis Child Fetal Neonatal Ed. 2008; 93(S1), Fa11.

33. Tang JWY, Dwyer JP, Rajesh U. Impact of introducing consultant resident on-call in a District General Hospital. J Obstet Gynaecol. 2012; 32: 736-9. doi: $10.3109 / 01443615.2012 .717992$

34. Woodhead N, Lindow S. Time of birth and delivery outcomes: a retrospective cohort study. J Obstet Gynaecol. 2012; 32: 335-7.

35. Woods CFG, Manohar S, Lindow SW. Obstetric consultant weekend on-call shift patterns have no effect on the management of spontaneous labour in a large maternity hospital. J Obstet Gynaecol. 2013; 33: 802-5.

36. Bragg F, Cromwell DA, Edozien LC, Gurol-Urganci I, Mahmood TA, Templeton A, et al. Variation in rates of caesarean section among English NHS trusts after accounting for maternal and clinical risk: cross sectional study. BMJ. 2010; 341: c5065. doi: 10.1136/bmj.c5065

37. Al-Suhel R, Gill S, Robson S, Shadbolt B. Kjelland's forceps in the new millennium. Maternal and neonatal outcomes of attempted rotational forceps delivery. Aust N Z J Obstet Gynaecol. 2009; 49: 510-4. doi: 10.1111/j.1479-828X.2009.01060.x. 
38. Tempest N, Hart A, Walkinshaw S, Hapangama DK. A re-evaluation of the role of rotational forceps: retrospective comparison of maternal and perinatal outcomes following different methods of birth for malposition in the second stage of labour. BJOG. 2013; 120: 1277-84. doi: 10.1111/1471-0528.12199

39. Aiken CE, Park H, Brockelsby J, Prentice A. Operative skills training in obstetrics identification of increased training needs for junior doctors. Arch Dis Child Fetal Neonatal Ed. 2014; 99: A17. doi: 10.1136/archdischild-2014-306576.47

40. Manktelow BM, Smith LK, Evans TA, Hyman-Taylor P, Kurinczuk JJ, Field DJ, et al. Perinatal Mortality Surveillance Report: UK Perinatal Deaths for births from January to December 2013. Leicester: The Infant Mortality and Morbidity Group, Department of Health Sciences, University of Leicester, 2015.

41. Sandall J, Soltani H, Gates S, Shennan A, Devane D. Midwife-led continuity models versus other models of care for childbearing women. Cochrane Database of Systematic Reviews. 2013; 8: CD004667. doi: 10.1002/14651858.CD004667.pub3

42. Parliamentary Office of Science and Technology. Caesarean sections - Postnote no. 184. London: Parliamentary Office of Science and Technology, 2002.

43. National Health Service Litigation Authority. Ten years of maternity claims: an analysis of NHS litigation authority data. London: National Health Service Litigation Authority, 2012.

44. Weindling AM. The confidential enquiry into maternal and child health (CEMACH). Arch Dis Child. 2003; 88: 1034-37. doi: 10.1136/adc.88.12.1034 (CEMACH). Arch Dis Child. 2003; 88: 1034-37. doi: 10.1136/adc.88.12.103 


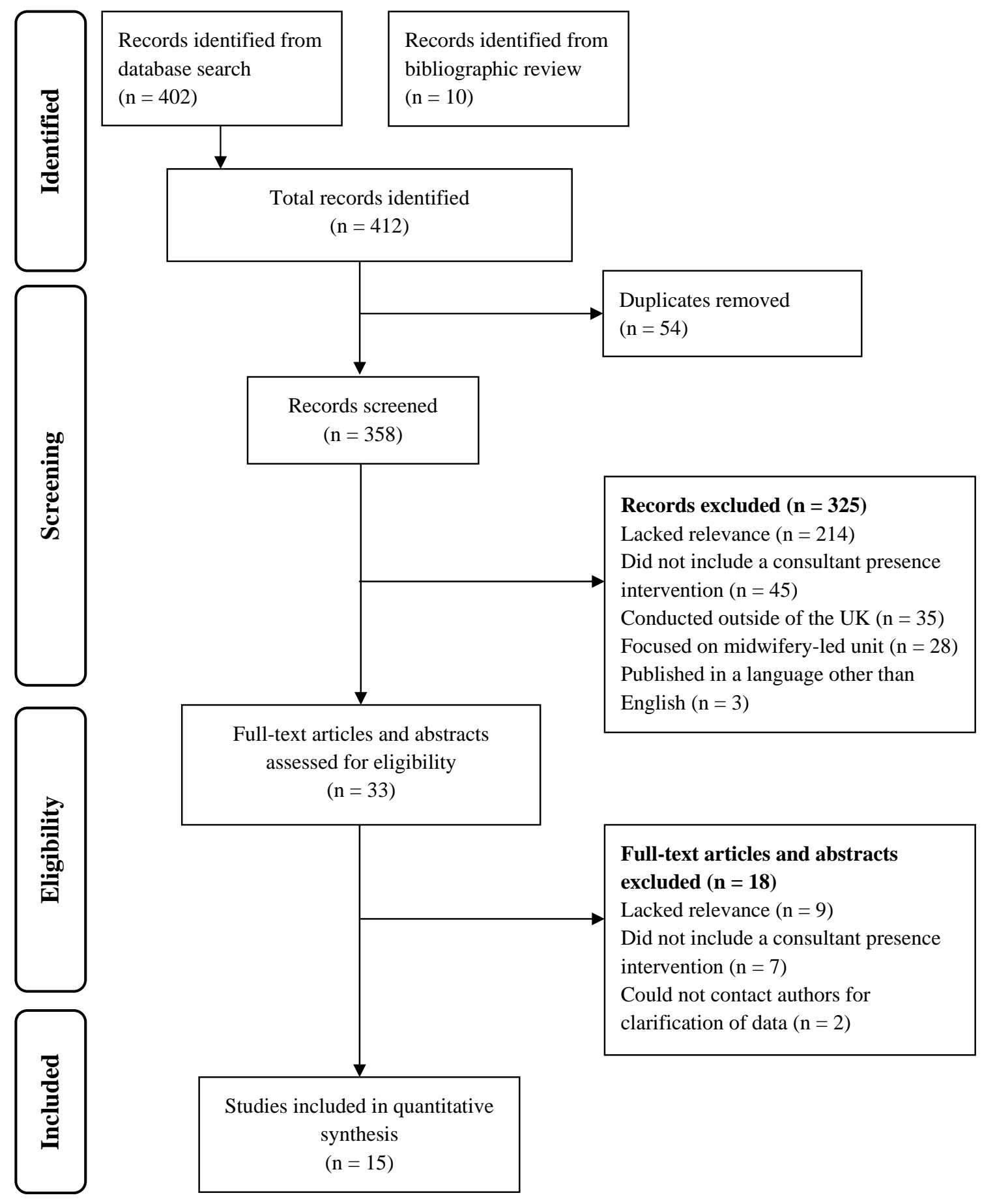

Figure 1. Flow chart of screening and selection of studies. 
Table 1. Meta-analysis of all outcomes

\begin{tabular}{|c|c|c|c|c|c|c|c|c|c|c|}
\hline \multicolumn{6}{|c|}{ Overall meta-analysis } & \multicolumn{3}{|c|}{ Type of increase in Consultant Presence } & \multicolumn{2}{|c|}{ Type of unit } \\
\hline Outcome & $\begin{array}{l}\text { No. of } \\
\text { studies }\end{array}$ & $\begin{array}{l}\text { No. of } \\
\text { births }\end{array}$ & $\begin{array}{l}\text { No. of women } \\
\text { during } \\
\text { increased } \\
\text { consultant } \\
\text { presence }(\%)\end{array}$ & $I^{2}(\%)$ & $\begin{array}{l}\text { Overall OR } \\
(95 \% \text { CI) }\end{array}$ & $\begin{array}{l}\text { Group } 1 \text { OR } \\
(95 \% \mathrm{CI})\end{array}$ & $\begin{array}{l}\text { Group } 2 \text { OR } \\
(95 \% \text { CI })\end{array}$ & $\begin{array}{l}\text { Group } 3 \text { OR } \\
(95 \% \text { CI) }\end{array}$ & $\begin{array}{l}\text { Secondary OR } \\
(95 \% \text { CI) }\end{array}$ & $\begin{array}{l}\text { Tertiary OR } \\
(95 \% \text { CI) }\end{array}$ \\
\hline $\begin{array}{l}\text { Emergency } \\
\text { caesarean section }\end{array}$ & 14 & 119397 & $64285(53.8)$ & 68.2 & $0.98(0.92-1.05)$ & $0.99(0.94-1.05)$ & $0.91(0.86-0.96)$ & $1.01(0.87-1.17)$ & $0.95(0.88-1.05)$ & $1.04(0.91-1.18)$ \\
\hline $\begin{array}{l}\text { Non-instrumental } \\
\text { vaginal delivery }\end{array}$ & 14 & 117686 & $64773(55.0)$ & 71.7 & $1.00(0.95-1.06)$ & $0.99(0.94-1.03)$ & $1.07(1.02-1.12)$ & $0.97(0.87-1.10)$ & $1.03(0.96-1.10)$ & $0.95(0.85-1.07)$ \\
\hline $\begin{array}{l}\text { Instrumental } \\
\text { delivery }\end{array}$ & 14 & 117686 & $64773(55.0)$ & 49.2 & $1.04(0.98-1.10)$ & $1.04(0.98-1.10)$ & $1.01(0.90-1.14)$ & $1.06(0.95-1.19)$ & $1.05(0.97-1.14)$ & $1.02(0.91-1.13)$ \\
\hline NICU admission & 7 & 52517 & $25268(48.1)$ & 62.1 & $1.03(0.87-1.23)$ & $0.93(0.80-1.09)$ & $1.23(1.06-1.43)$ & $0.95(0.69-1.31)$ & & \\
\hline Neonatal death & 3 & 15090 & $5939(39.4)$ & 0.0 & $1.27(0.51-3.18)$ & & & & & \\
\hline Stillbirth & 4 & 36860 & $16335(44.3)$ & 50.3 & $1.17(0.76-1.80)$ & & & & & \\
\hline PPH & 4 & 24564 & $12243(49.8)$ & 83.8 & $1.55(0.72-3.33)$ & & & & & \\
\hline $\begin{array}{l}3^{\text {rd }} \text { and } 4^{\text {th }} \text { degree } \\
\text { tears }\end{array}$ & 4 & 24220 & 11 811(48.8) & 0.0 & $1.09(0.90-1.32)$ & & & & & \\
\hline
\end{tabular}

Group 1 = hours of rostered consultant presence per day during the weekend vs. hours of rostered consultant presence per day during the week, Group 2 = hours per week of rostered consultant presence pre-increase vs. hours per week of rostered consultant presence post increase, Group $3=$ no rostered consultant presence vs. rostered consultant presence 


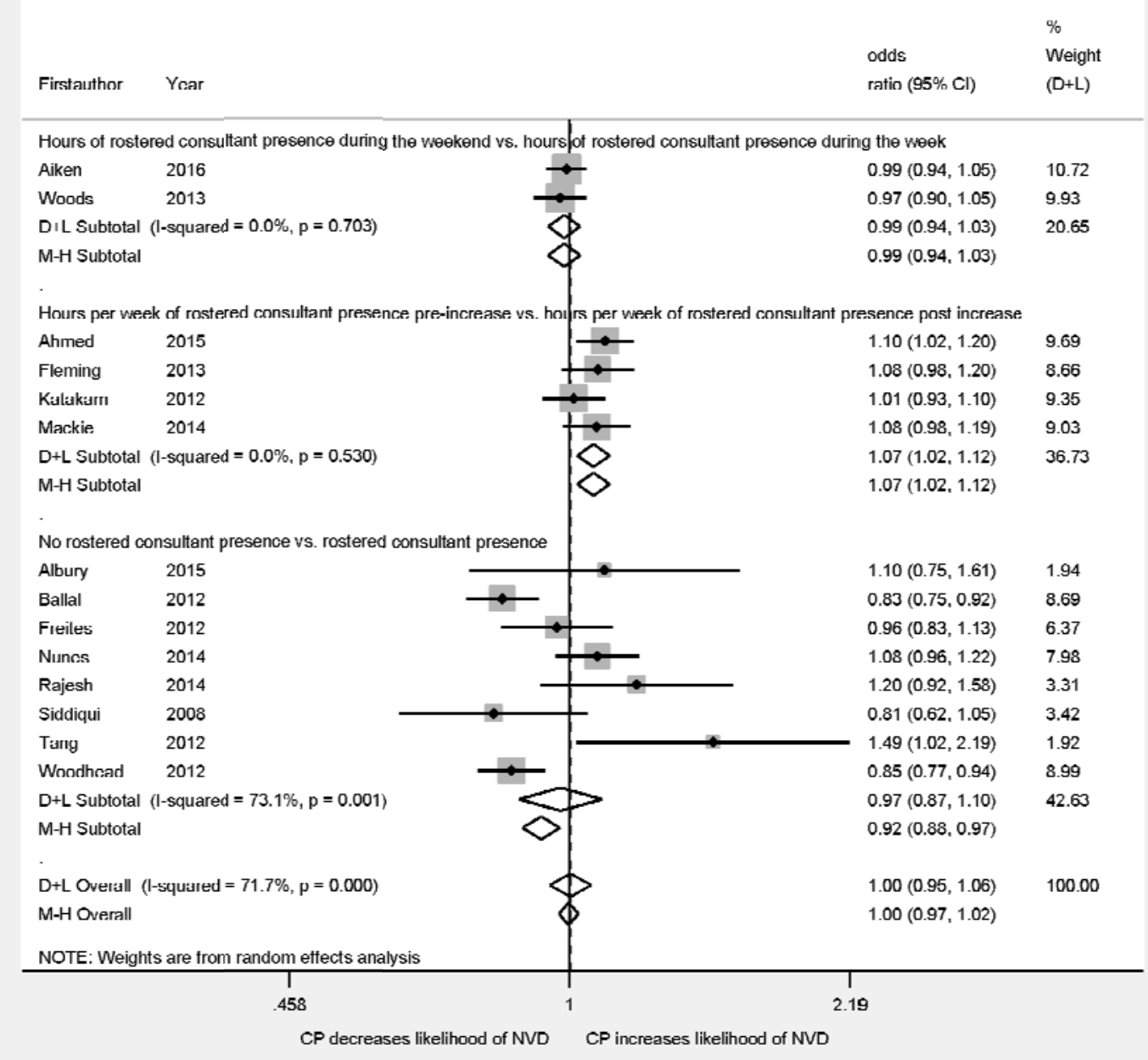

Figure 2. Forest plot of odds ratios of non-instrumental vaginal deliveries stratified by comparison group using random effects analysis 


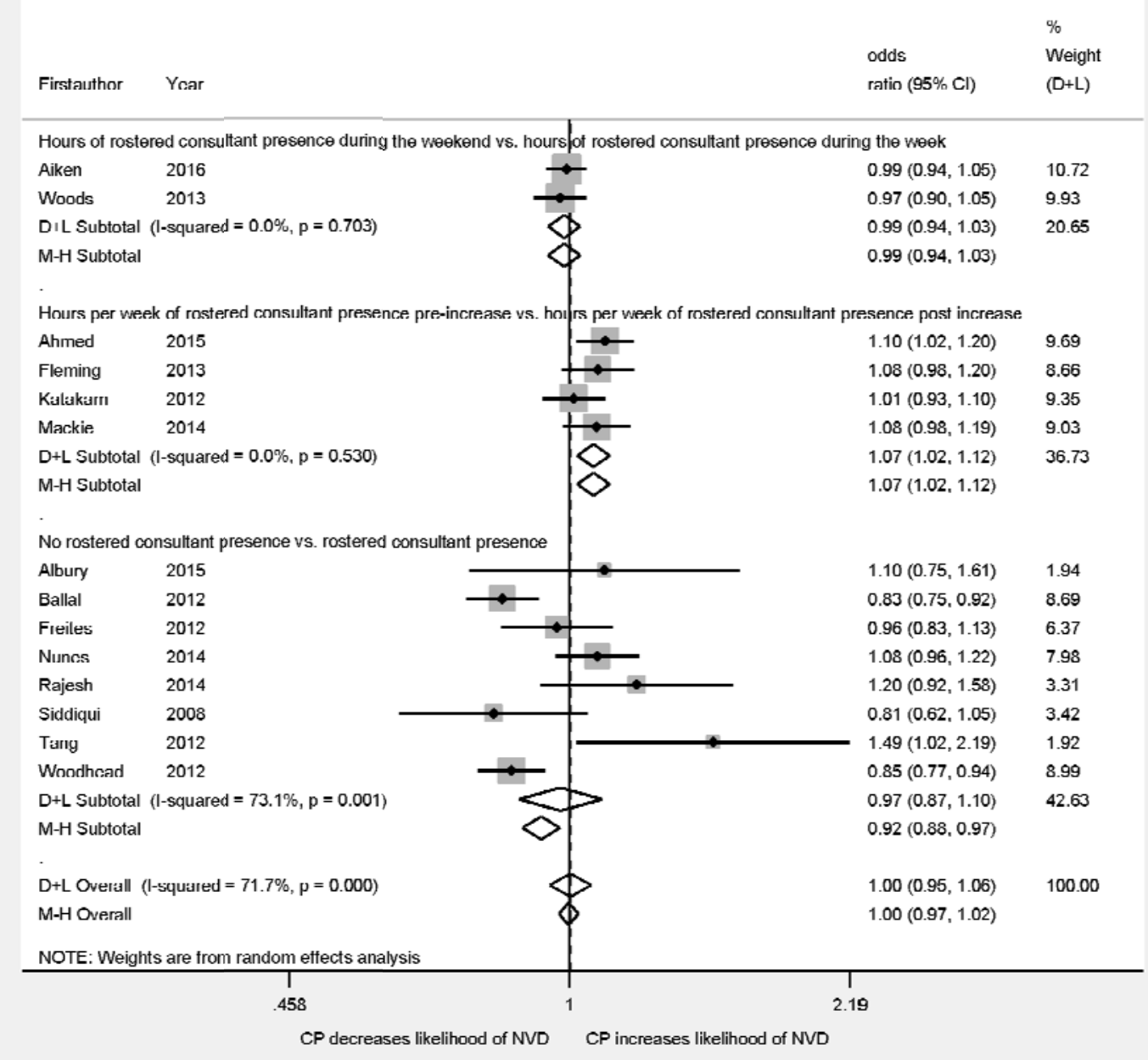

Figure 3. Forest plot of odds ratios of non-instrumental vaginal deliveries stratified by comparison group weighted from random effects analysis 